\title{
ANÁLISIS DOCUMENTAL BIBLIOGRÁFICO. OBTENIENDO EL MÁXIMO RENDIMIENTO A LA REVISIÓN DE LA LITERATURA EN INVESTIGACIONES CUALITATIVAS
}

\author{
Antoni Casasempere-Satorres ${ }^{1}$ y María Luisa Vercher-Ferrándiz ${ }^{2}$ \\ CualSoft Consultores, España. antoni@cualsoft.com \\ 2Universidad Politécnica de Valencia, España. maverfer@upv.es
}

\begin{abstract}
Resumen. El proceso de revisión de la literatura es una parte compleja de las investigaciones cualitativas, cuantitativas y mixtas desarrollándose a lo largo de toda la investigación, desde la formulación del problema del estudio, pasando por la elaboración de un marco conceptual hasta el desarrollo de la discusión o diálogo teorético. El objetivo del presente trabajo se centra en ofrecer un procedimiento innovador que facilita una revisión sistemática de la literatura en investigaciones cualitativas basada en un proceso analítico metodológicamente estructurado, análisis documental, y apoyado por las posibilidades técnicas que ofrecen las herramientas informáticas como Mendeley o MAXQDA. El procedimiento metodológico de Análisis Documental Bibliográfico sirve como soporte al desarrollo del propósito de la investigación al establecer los límites investigadores del estudio, propicia la elaboración de un marco conceptual al permitir la articulación de indicadores a partir de conceptos y facilita el desarrollo de la discusión del estudio o diálogo teorético. El resultado es la fluida integración del material documental en una base de datos que sirve de apoyo a las paráfrasis resultantes siendo ésta una estrategia para resumir datos y elaborar categorías analíticas. El procedimiento se ha probado exitosamente en diferentes investigaciones aportando una mayor claridad al proceso de revisión de la literatura en investigaciones cualitativas.
\end{abstract}

Palabras clave: Revisión Literatura; Análisis Documental; Mendeley; MAXQDA; Paráfrasis.

\section{BIBLIOGRAPHIC DOCUMENTARY ANALYSIS. GETTING THE MOST OUT OF THE LITERATURE REVIEW IN QUALITATIVE RESEARCH}

\begin{abstract}
The review process of the literature is a complex part of qualitative, quantitative and mixed methods research, developed throughout the entire investigation, from the formulation of the study problem, through the improvement of a conceptual framework to the rise of the discussion or theoretical dialogue. The objective of this paper is to offer an innovative procedure that facilitates a systematic review of the literature in qualitative research based on a methodologically structured analytical process, documentary analysis, and supported by the technical possibilities offered by computer tools such as Mendeley or MAXQDA. The methodological procedure of the Bibliographic Documentary Analysis includes the support to the development of the purpose of the investigation by establishing the investigative limits of the study, facilitating the elaboration of a conceptual framework by allowing the articulation of indicators based on concepts and the development of the study discussion or theoretical dialogue. The result is the smooth integration of documentary material into a database with the support of paraphrase, strategy to summarize data and develop analytical categories. The procedure has been successfully tested in different investigations providing greater clarity to the process of reviewing the literature in qualitative research.
\end{abstract}

Keywords: Literature Review; Documentary Analysis; Mendeley; MAXQDA; Paraphrases.

\section{INTRODUCCIÓN}

Tanto a investigadores/as noveles como veteranos/as les resulta complejo el proceso de la revisión bibliográfica de una investigación, ya sea cualitativa, cuantitativa o mixta (Onwuegbuzie y Frels, 2015). El proceso de revisión de la literatura se desarrolla a lo largo 
de toda la investigación y especialmente en la concreción del propósito de la investigación, la elaboración de un marco conceptual y en la discusión final propiciando el diálogo teorético (Casasempere-Satorres y Vercher-Ferrándiz, 2017).

Las tecnologías informáticas disponibles permiten la sistematización y ayudan a estructurar el trabajo documental bibliográfico. El procedimiento denominado Análisis Documental Bibliográfico aúna el trabajo metodológico del análisis documental, como técnica de toma de datos en investigación cualitativa, con diferentes procedimientos informatizados con herramientas como Mendeley (www.mendeley.com) o MAXQDA (www.maxqda.com) para lograr que el proceso de revisión de la literatura sea más fluido, sencillo y comprehensivo.

En el presente trabajo se describen las principales etapas del Análisis Documental Bibliográfico y los procedimientos técnicos e informáticos asociados sugeridos a los y las investigadores/as cualitativos. Adicionalmente, se ejemplifica en detalle el proceso de uso de paráfrasis para crear un informe bibliográfico final a partir de corpus textuales extensos.

\section{EL ANÁLISIS DOCUMENTAL BIBLIOGRÁFICO EN EL DISEÑO DE LA INVESTIGACIÓN CUALITATIVA}

El procedimiento documental en las investigaciones cualitativas se ha desarrollado con profusión en la literatura especializada, por ejemplo en Cohen, Manion, y Morrison (2017), se puede encontrar el trabajo de Suri, capítulo 21 , en que se trata el meta-análisis, las revisiones sistemáticas y las síntesis de investigaciones y las etapas necesarias para llevarlo a cabo; en el trabajo de Lichtman (2006) se desarrollan seis etapas para realizar la revisión de la literatura o en el campo de las ciencias de la salud se puede citar como ejemplo el trabajo de Jones en Saks y Allsop (2007).

Al observar la Figura 1 (Newman y Gough, 2020), se han sombreado las etapas que se desarrollan en el presente trabajo. 


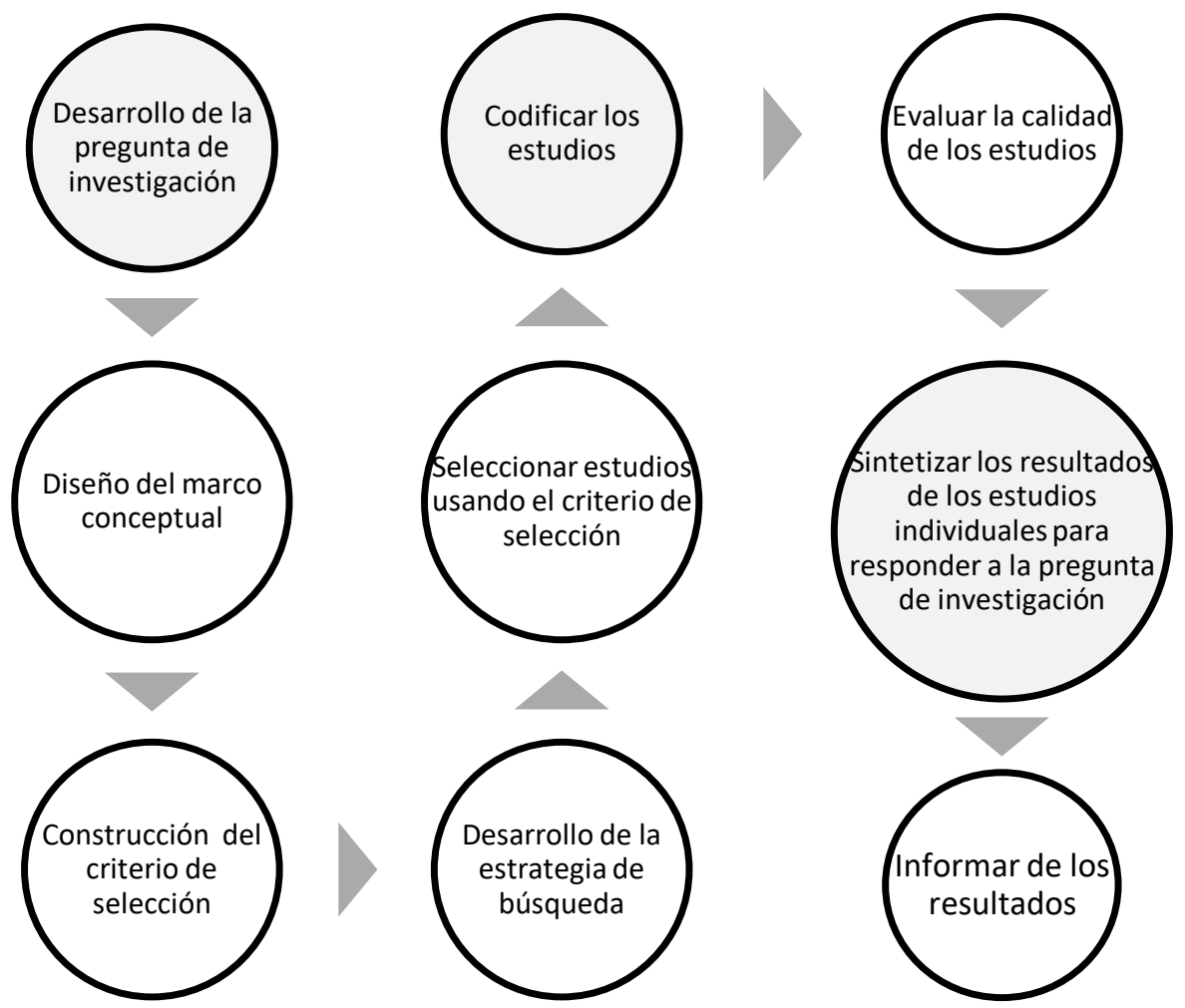

Figura 1. Proceso sistemático de la literatura.

Adaptado de Newman y Gough (2020:6)

El procedimiento descrito en Newman y Gough (2020) se basa en cálculos estadísticos centrados en medir el tamaño del efecto y su dirección aunque el procedimiento no sea adecuado para muchas revisiones sistemáticas de la literatura en metodología cualitativa que en muchos casos se basa en ordenar, seleccionar y organizar la información (Lichtman, 2006: 108). Sin embargo, de un modo más aplicado se encuentra descrito un procedimiento informatizado en el trabajo de Kuckartz y Rädiker (2019) que ofrece interesantes posibilidades y que con la presente propuesta se propone ampliar.

Seguidamente, se exponen los tres momentos fundamentales del proceso de Análisis Documental Bibliográfico: concreción del propósito de investigación, elaboración del marco conceptual y fomento del diálogo teorético.

\subsection{Concretando el propósito de investigación}

En el trabajo de Wolcott (2009), citado por Saldaña (2011), se constata la importancia de sintetizar en una frase el proceso investigador en un estudio cualitativo, el último 'por qué' conductor de la investigación. En anteriores trabajos (Casasempere-Satorres, 2016), se ha 
destacado la importancia de establecer un propósito de la investigación. Entre los componentes citados del propósito de la investigación, destaca la importancia de realizar la primera aproximación a la literatura con objeto de delimitar lo que se ha investigado al respecto del fenómeno de interés en la investigación en ciernes y a partir de qué punto se puede generar nuevo conocimiento.

En esta primera etapa y considerando que el propósito de la investigación contiene los principales conceptos del estudio, se realizarán búsquedas combinadas de conceptos en las bases de datos especializadas de cada disciplina con el objeto de discernir las principales lecturas que mejor tratan el tema propuesto para la investigación.

Es recomendable estructurar los conceptos en una tabla en la que se organicen en orden decreciente de mayor a menor relevancia al respecto del propósito de investigación. Las búsquedas combinadas de conceptos en bases de datos digitales especializadas darán como resultado diferentes hallazgos que procede valorar en términos de calidad y adecuación al propósito de la investigación. En este sentido, se sugiere que cada lectura evaluada como muy relevante se incorpore a un gestor bibliográfico, en nuestro caso Mendeley. Al tiempo que se introduce la referencia en el gestor bibliográfico, se comprueba su precisión en cuanto a estilo (APA en metodología cualitativa).

Las referencias destacadas se deben evaluar en profundidad y para ello se necesitará disponer del texto completo y no solo del resumen. Las referencias, normalmente en formato PDF se llevarán a la base de datos informatizada (MAXQDA) y se les añade una nota de documento que contiene una ficha de evaluación, Figura 2, al modo de las propuestas en la literatura (Smallbone y Quinton, 2011).

\begin{tabular}{|c|c|c|}
\hline Sistema de documentos & & T Memo de documento: Bosom, Fernández, et all (2007)-Excellence in virtual education \\
\hline Documentos & & 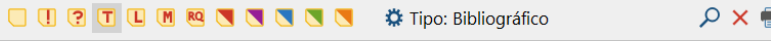 \\
\hline $\begin{array}{l}\text { - } \text { Adamus, Getto, et all (2009)-Gender and E-Tutoring - A concept } \\
\text { - B Bennett, Marsh (2010)-Are We Expecting Online Tutors }\end{array}$ & & 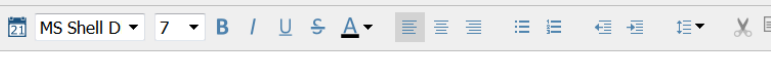 \\
\hline 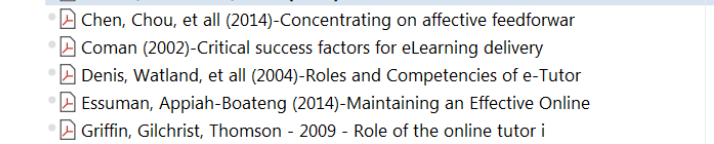 & & $\begin{array}{l}\text { Estilo } \\
\text { ¿Está construido claramente? } \\
\text { ¿Es un documento de opinión, artículo, documento empírico o una revisión sistemática? } \\
\text { ¿Sstán bien definidas las palabras clave? argumento dentro de un desarrollo lógico? } \\
\text { ¿Añaden valor las tablas y diaaramas a la explicación o a las conclusiones? }\end{array}$ \\
\hline
\end{tabular}

Figura 2. Ejemplo de ficha bibliográfica en memo de documento en MAXQDA 2020. 
Adicionalmente, se pueden insertar las referencias bibliográficas de interés desde el programa Mendeley al programa MAXQDA simplemente habiéndolas guardado en formato RIS (Research Information Systems) desde el propio menú Importar (Figura 3). Este sistema permite incorporar al programa los metadatos de Mendeley y el archivo del documento en PDF si se encuentra disponible.

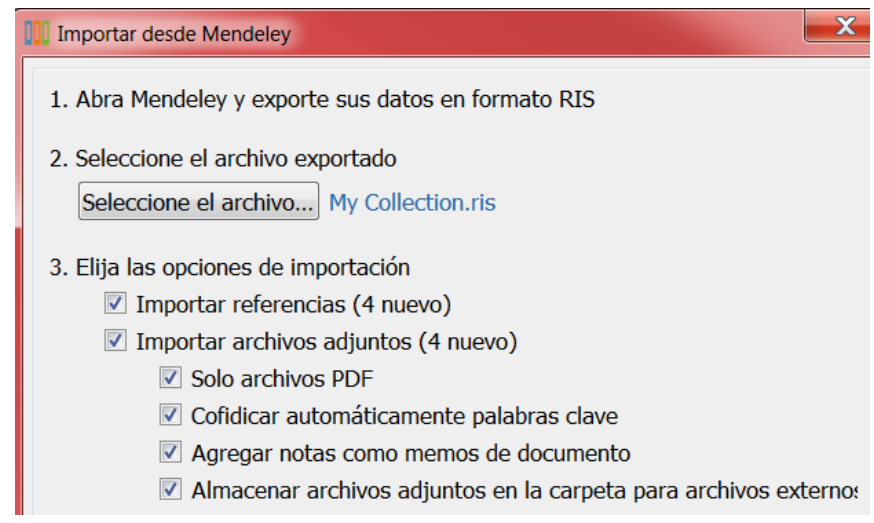

Figura 3. Importando referencias de Mendeley a MAXQDA 2020.

Este procedimiento ha ayudado a concretar el propósito de investigación y a establecer cuál es el límite entre lo investigado en el campo de interés y a partir de qué punto es posible generar conocimiento. Si el trabajo investigador que se afronta dispone de conceptos adecuadamente investigados en la literatura, el paso siguiente será concretar una tabla en la que se articulen y hagan operativos los indicadores de dichos constructos, es el momento de iniciar el desarrollo del marco conceptual.

\subsection{Elaborando el marco conceptual}

El marco conceptual deductivo es un diagrama destacado en la mayoría de investigaciones (Imenda, 2014; Maxwell, 2009; Casasempere-Satorres, 2016). Aunque en el pasado se han defendido posturas alejadas de esta primera revisión bibliográfica, como la Teoría Fundamentada de Glaser y Strauss (1967), pronto se evidenció la relevancia de la sensibilidad teorética del investigador/a y la necesidad de un trabajo bibliográfico (Kelle, 2005).

Tras las primeras etapas del Análisis Documental Bibliográfico (Casasempere-Satorres y Vercher-Ferrándiz, 2017) en las que se ha contribuido a definir el propósito del estudio, se ha establecido el diseño del análisis sistemático bibliográfico y la búsqueda de la literatura mediante un sistema articulado de conceptos, se procede a utilizar las herramientas léxicas 
y de análisis de contenido cuantitativo (Kuckartz y Rädiker, 2019) que MAXQDA ofrece para aprehender los conceptos clave y los indicadores que los conforman en el corpus textual organizados en las pertinentes categorías.

La paráfrasis es una estrategia para resumir datos (Figura 4) y elaborar categorías analíticas (Kuckartz y Rädiker, 2019). Aunque las paráfrasis no requieren que los datos hayan sido codificados previamente, este proceso sí que contribuye a la generación inductiva de categorías analíticas (Kuckartz, 2014). En este trabajo se aplican las paráfrasis dentro de un contexto de Análisis Documental Bibliográfico (Casasempere-Satorres y Vercher-Ferrándiz, 2017) como eje central del proceso sistemático de documentación bibliográfica y apoyo a la generación de un marco conceptual que permita la adecuada toma de datos y su posterior análisis, tanto en estudios cualitativos como mixtos o cuantitativos.
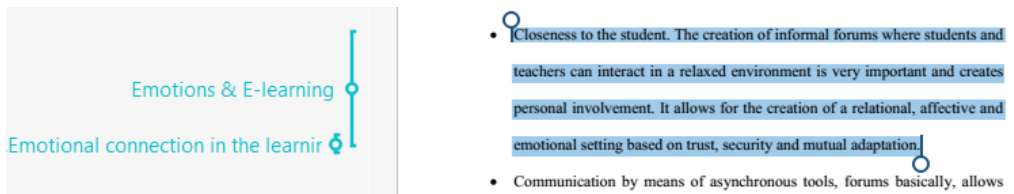

Informal forums promote personal $X$ involvement in students and improve mutual adaptation and security to the course (Bosom et al., 2007)

Figura 4. Parafraseando en MAXQDA 2020.

En especial, se presenta la estrategia de la utilización de las Combinaciones de palabras y la búsqueda dentro de los resultados encontrados para posteriormente y dentro del mismo proceso, proceder a parafrasear el hallazgo bibliográfico relevante para los objetivos del Análisis Documental Bibliográfico e incluso codificando manualmente el segmento de la literatura.

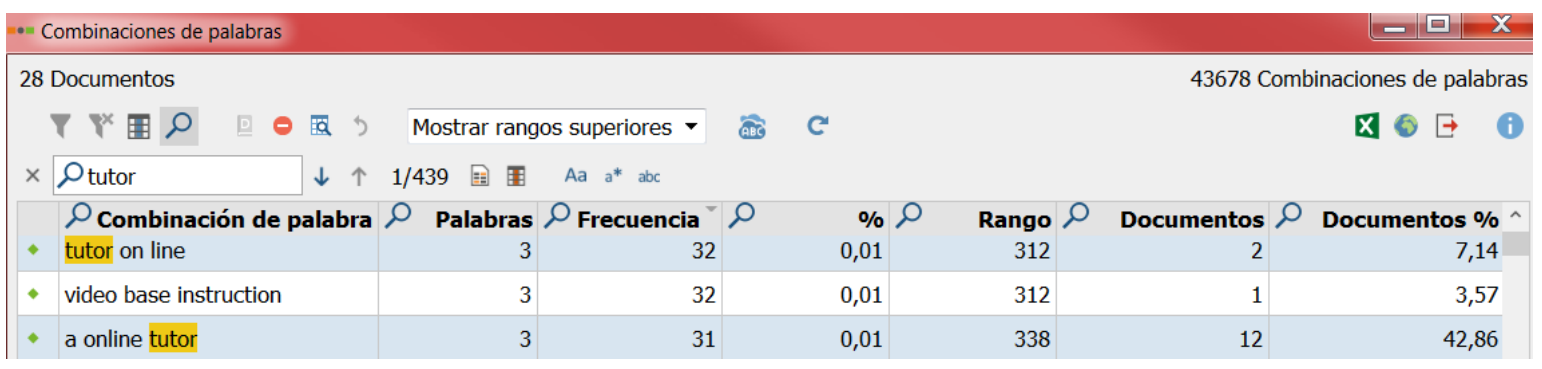

Figura. 5. Combinaciones de palabras en MAXQDA 2020.

Este tránsito simultáneo entre el procedimiento automático y el manual permite, en un espacio breve de tiempo, crear un listado de indicadores que conformarán el futuro marco conceptual. Adicionalmente, se dispone de una colección de paráfrasis sobre los pasajes relevantes que en el futuro se trasladarán a la memoria o informe final conformando, por 
ejemplo, el diálogo teorético de la investigación. Las paráfrasis realizadas deben ser breves y no perder el significado primario de la idea trasladada por el autor de la obra. Adicionalmente, se incluirá al final de la misma y entre paréntesis los apellidos de los autores y año de la obra parafraseada, aunque este punto se puede obviar si los documentos bibliográficos se han organizado por este criterio ya que la categorización de las paráfrasis permitirá la ubicación precisa de cada texto junto a la obra original.

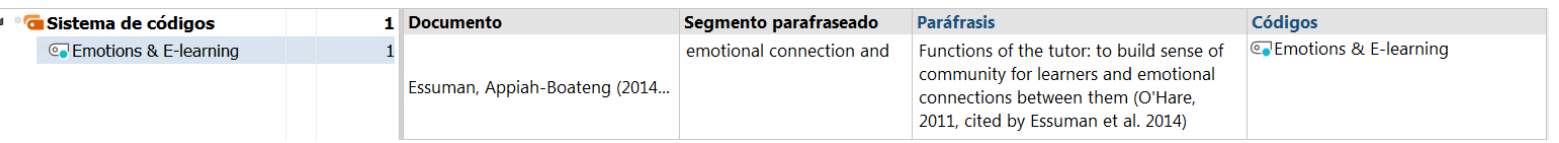

Figura 6. Herramienta de categorización de paráfrasis en MAXQDA 2020.

Una estrategia que ha resultado eficiente para elaborar artículos académicos es crear una estructura de indicadores (códigos) dentro de la herramienta informática con la misma denominación que las partes de un artículo (Introducción, Contexto teórico, Metodología, etc.) y sus epígrafes. Con estos indicadores se han codificado las paráfrasis para determinar la posición en la que se usarán en la narrativa que terminará en comunicación académica. Si dentro de cada apartado del artículo procede incluir varias paráfrasis, se procede iniciando la paráfrasis con un número que permite ordenarlas y saber el orden en el que va cada fragmento de la literatura revisada. La Figura 7 muestra un ejemplo en el que se incluye la estructura del futuro artículo en forma de códigos, el documento original y su segmento junto con la paráfrasis a usar en el artículo y el código instrumental asociado.

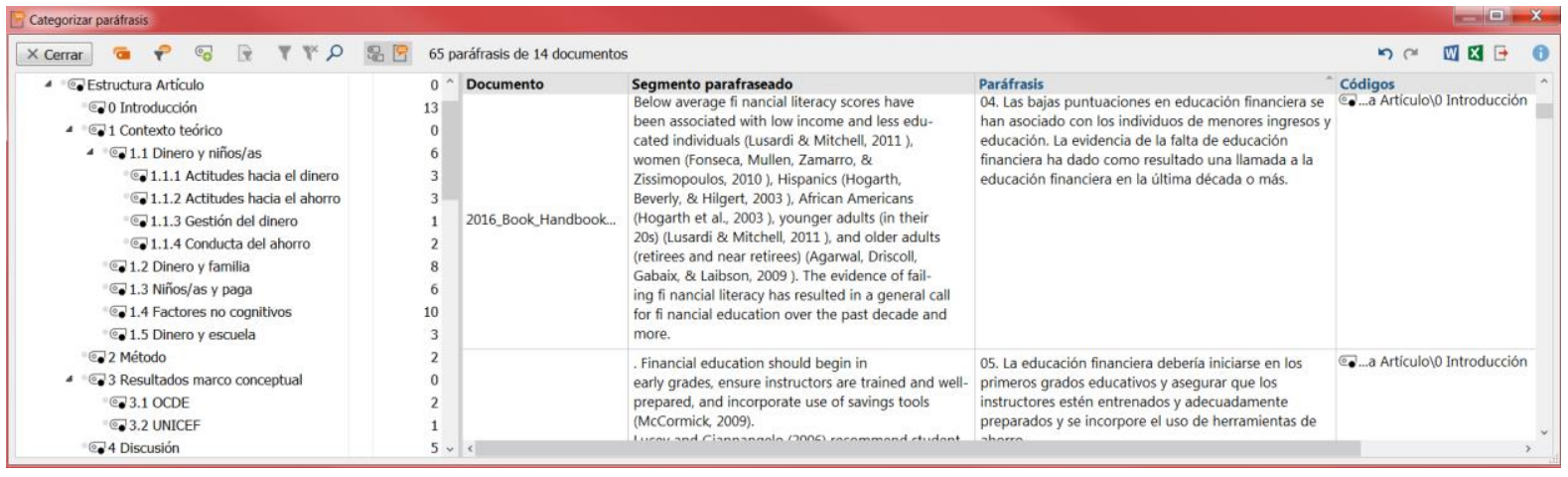

Figura 7. Contexto de trabajo con códigos instrumentales y paráfrasis.

El programa MAXQDA proporciona interesantes herramientas analíticas avanzadas para desarrollar el procedimiento anteriormente descrito como Categorizar paráfrasis o la Matriz de paráfrasis. En la herramienta de categorización de paráfrasis se pueden revisar y 
organizar las paráfrasis, incluyen la creación de nuevos códigos que representen a indicadores del marco conceptual futuro. La combinación de las paráfrasis en la herramienta de categorización de paráfrasis, una vez listada o impresa, puede muy bien conformar el núcleo de la narrativa de la revisión bibliográfica sistemática realizada y contribuir al desarrollo del futuro diálogo teorético al haber anclado los conceptos clave mediante códigos o indicadores.

La matriz de paráfrasis ha servido de utilidad en nuestros trabajos al permitir comparar partes del corpus textual, por ejemplo entre autores o corrientes teóricas (Figura 8).

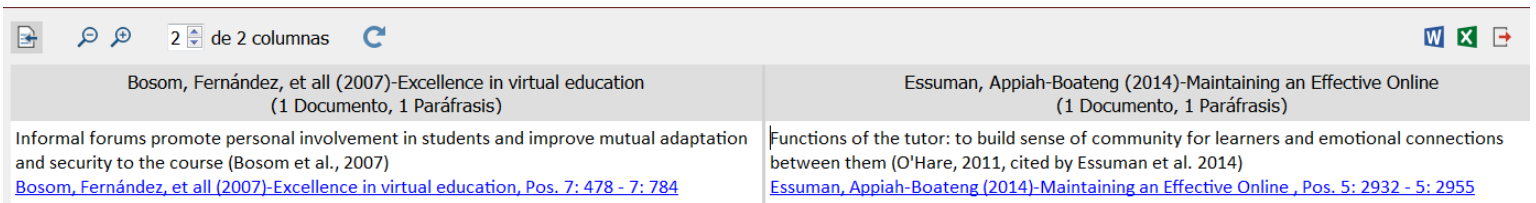

Figura 8. La matriz de paráfrasis en MAXQDA 2020.

\subsection{Propiciando el diálogo teorético}

La etapa final de la investigación es elaborar la discusión a partir del contraste entre los resultados obtenidos en el estudio presente y las conclusiones de los estudios considerados en el Análisis Documental Bibliográfico.

Un método pertinente puede ser utilizar las variables de documento o código aplicado a la literatura que se ha manejado, una variable de documento aplica un único valor al caso y la variable de código puede aplicar diferentes valores de variable a los códigos o etiquetas contenidos en un único documento. De este modo, se puede crear la variable 'resultados' con los valores: critica, apoya, etc. para etiquetar fragmentos de la literatura y recuperar aquellos autores/as que convergen o no lo hacen con nuestro trabajo al respecto de un tema concreto codificado cualitativamente. 


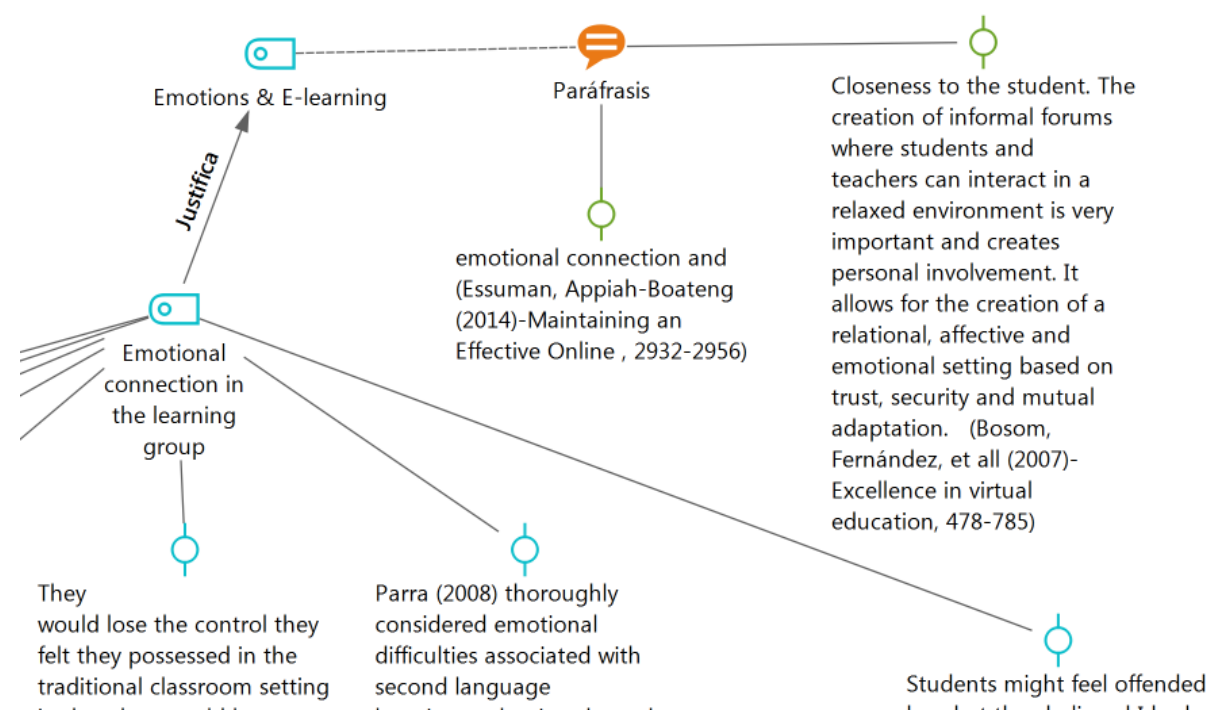

Figura 9. Apoyando el diálogo teorético con MAXQDA 2020.

Adicionalmente, se pueden trazar vínculos entre nuestros datos o resultados y las obras analizadas documentalmente (Figura 9), creando de este modo una trama de relaciones que permite de un modo sencillo trazar el diálogo teorético. Esta red se puede representar en el escenario gráfico de MAXQDA, MAXMapas, permitiendo seguir las interrelaciones de las ideas de la literatura y nuestros resultados.

\section{CONCLUSIONES}

Se ha expuesto en este trabajo la importancia de la revisión sistemática de la literatura a través de la aplicación de herramientas informáticas ya que facilitan la revisión eficiente de grandes volúmenes de documentos pues, dirigen la atención del investigador a los segmentos de interés real al propósito de la investigación ayudándole en las fases de delimitación del propósito, la construcción del marco conceptual y el desarrollo del diálogo teorético, permitiendo además, que durante el proceso pueda aprehender, registrar, codificar y parafrasear las ideas relevantes que van surgiendo y en último lugar relacionarlas y generar el diálogo teorético.

En el trabajo de Woods, Paulus, Atkins, y Macklin (2015) se constata el aumento de investigadores/as en todo el mundo que utilizan programas QDA (Qualitative Data Analysis) para llevar a cabo sus trabajos analíticos aunque con una infrautilización de las posibilidades de estas herramientas. En el anterior trabajo, contrastando usuarios de NVivo y ATLAS.ti, se evidenciaba la necesidad de obtener un mejor aprovechamiento de las herramientas informáticas, a pesar de la existencia de trabajos en la materia, por ejemplo para ATLAS.ti 
(Friese, 2019; Woolf \& Silver, 2017a) o para NVivo (O’Neill, Booth, y Lamb, 2018; Woolf y Silver, 2017b).

El presente trabajo expone en detalle el componente interno del Análisis Documental Bibliográfico (Casasempere-Satorres y Vercher-Ferrándiz, 2017) basado en la combinación de herramientas analíticas automáticas de MAXQDA y el trabajo analítico manual que las paráfrasis y su categorización posibilitan, sin dejar de lado, si procede, el proceso tradicional de codificación manual de la literatura utilizada en la investigación.

El objetivo fundamental de la presente propuesta era evidenciar la efectividad del uso de herramientas informáticas en la revisión bibliográfica y no solo en el análisis de los datos resultantes de la investigación, pues facilita la revisión documental de ingentes cantidades de documentos, de manera eficiente con el considerable ahorro de tiempo y mantiene un registro accesible y relacionable de todas las ideas que se han ido aprehendiendo durante el proceso, permitiendo tanto a investigadores/as noveles como consolidados/as la recuperación e interrelación de las mismas en cada fase de la investigación. Este sistema ha sido probado satisfactoriamente en investigaciones sociales, de la conducta y ciencias de la salud reduciendo el tiempo empleado en la revisión bibliográfica considerablemente.

\section{REFERENCIAS}

Casasempere-Satorres, A. (2016). El mapa del diseño: una herramienta heurística para trazar el flujo metodológico y resolver eficientemente una investigación con metodología cualitativa o mixta. En Congreso Español de Sociología" Grandes transformaciones sociales, nuevos desafíos para la sociología" (p. 28). Gijón: Federación Española de Sociología.

Casasempere-Satorres, A., \& Vercher-Ferrándiz, M. L. (2017). Bibliographical documentary analysis with MAXQDA. En Marburger Arbeitsgruppe für Methoden \& Evaluation. Berlín: MAGMA.

Cohen, L., Manion, L., \& Morrison, K. (2017). Research methods in education (Octava edi). Abingdon, Reino Unido: Routledge.

Friese, S. (2019). Qualitative Data Analysis with ATLAS.ti. Qualitative Research. Sage Publishing. https://doi.org/10.1177/1468794113475420

Glaser, B. G., \& Strauss, A. L. (1967). The Discovery of Grounded Theory: Strategies for Qualitative Research. New Jersey: Aldine Transaction. Recuperado de https://books.google.com/books?id=oUxEAQAAIAAJ\&pgis=1

Imenda, S. (2014). Is there a conceptual difference between theoretical and conceptual frameworks? Journal of Social Sciences, 38(2), 185-195. https://doi.org/10.1080/09718923.2014.11893249

Kelle, U. (2005). «Emergence» vs. «forcing» of empirical data? A crucial problem of «Grounded Theory» reconsidered. Forum: qualitative social research, 6(2). Recuperado de http://www.qualitativeresearch.net/index.php/fqs/article/viewArticle/467/1000 
Kuckartz, U. (2014). Qualitative text analysis: A guide to methods, practice and using software. Londres: Sage Publishing.

Kuckartz, U., \& Rädiker, S. (2019). Analyzing qualitative data with MAXQDA. Text, audio, and video. Cham, Switzerland: Springer International Publishing. https://doi.org/10.1007/978-3-030-15671-8_1

Lichtman, M. (2006). Qualitative Research in Education: A User's Guide. Thousand Oaks, California: Sage Publications.

Maxwell, J. A. (2009). Designing a Qualitative Study. En The SAGE Handbook of Applied Social Research Methods (pp. 214-253). Londres: Sage Publications.

Newman, M., \& Gough, D. (2020). Systematic Reviews in Educational Research: Methodology, Perspectives and Application. En Systematic Reviews in Educational Research (pp. 3-22). Springer Fachmedien Wiesbaden. https://doi.org/10.1007/978-3-658-27602-7_1

O’Neill, M., Booth, S., \& Lamb, J. (2018). Using NVivoTM for literature reviews: The eight step pedagogy (N7+ 1). Recuperado de https://ro.ecu.edu.au/ecuworkspost2013/5505/

Onwuegbuzie, A. J., \& Frels, R. K. (2015). Using Q Methodology in the Literature Review Process: A Mixed Research Approach. Journal of Educational Issues, 1(2). https://doi.org/10.5296/jei.v1i2.8396

Saks, M., \& Allsop, J. (2007). Researching health: Qualitative, quantitative and mixed methods. Londres: Sage.

Saldaña, J. (2011). Fundamentals of Qualitative Research. Nueva York: Oxford University Press.

Smallbone, T., \& Quinton, S. (2011). A three-stage framework for teaching literature reviews: A new approach. The international journal of management education, 9(4), 1.

Woods, M., Paulus, T., Atkins, D. P., \& Macklin, R. (2015). Advancing Qualitative Research Using Qualitative Data Analysis Software (QDAS)? Reviewing Potential Versus Practice in Published Studies using ATLAS.ti and NVivo, 1994-2013. Social Science Computer Review, 34(5), 597-617. https://doi.org/10.1177/0894439315596311

Woolf, N. H., \& Silver, C. (2017a). Case Illustrations. Qualitative Analysis Using NVivo: The Five-Level QDA Method. https://doi.org/10.4324/9781315181660

Woolf, N. H., \& Silver, C. (2017b). Qualitative analysis using Nvivo: The five-level QDA® method. Qualitative Analysis Using NVivo: The Five-Level QDA Method. https://doi.org/10.4324/9781315181660 